wenn unter Aufstützen der Hand in der Hüfte rollende Bewegungen ausgeführt werden. Das Geräusch ist am inneren Rand zu lokalisieren. Da Pat. sich nur über sein Leiden unterrichten will, verzichtet er auf eine Behandlung. - Nachuntersuchung im Juli I922 ergibt keine Veränderung.

3. 3Ijährige Arzt K. F. Keine Tuberkulose in der Vorgeschichte. Seit I 2 Jahren ein nicht schmerzhaftes Knacken an beiden Schulterblättern, links aber bedeutend ausgesprochener als rechts. - Befund am I9. VI. I922: Organgesunder Mann in sehr gutem Ernährungszustand. Beträchtliche Skoliose im Fals-Brustabschnitt mit linksseitigem Rippenbuckel. Links starkes Knacken bei auf- und abgleitenden Bewegungen des Schulterblattes. Keine Schmerzen, nur ein unangenehmes Gefüh1.

4. 46jährige Ehefrau Fr. G., 2 Kinder, mehrere Fehl- und Frühgeburten. Seit 2 Jahren Nackensteifigkeit und seit April 1922 Knacken in der rechten Schulter und nächtliche Schmerzen. Befund am I 5. VII. I922. Große gesunde Frau mit leichtem, rechtem Rippenbuckel, der vom Vater ererbt sein soll. Fïhl- und hörbares Knacken des rechten Schulterblatts. Pat. steht noch in Behandlung.

Die drei letzten Patienten, der eine ohne, zwei mit Schmerzen, lehnten einen operativen Eingriff $\mathrm{ab}$, der mir aber dort, wo größere Beschwerden bestehen, angezeigt erscheint, zumal da bisher alle operierten Fälle der Literatur geheilt wurden und sich Rückfälle nirgends beschrieben finden. Der Eingriff besteht in Abmeißeln oder Abkneifen etwaiger Knochenvorsprünge von einem Längsschnitt am inneren Schulterblattrand oder von einem Bogenschnitt am inneren oberen Wínkel aus. Auch Muskelplastiken sind von LotHEISSEN (zugleich mit Entfernung des gewulsteten Randes) und MAUCLAIRE gemacht worden, haben aber sicher den Nachteil, zu Rückfällen führen zu können, während eine Bandagenbehandlung nur bei messerscheuen Patienten zu empfehlen ist.

Zusammenfassend sei also gesagt, daß das Schulterblattkrachen vor allem durch Veränderungen am Knochengerüst (Exostosen usw.) hervorgerufen wird, und daß die Befunde an den Muskeln (Schwielenbildung) und das Auftreten von Schleimbeuteln meist als sekundär anzusehen sind. Damit die häufig. geringen Anomalien zu einem störenden und schmerzhaften Krachen führen, müssen gewisse Vorbedingungen durch Skoliose, Traumen, Tuberkulose usw, erfüllt sein. Die Behandlung ist operativ, die Prognose günstig.

\section{ÜBER GEFAHREN BEI DER ANWENDUNG DER HYPOPHYSENPRÄPARATE.}

Von

Dr. Max SAMUEL in Köln a. Rh.

In der "Therapie der Gegenwart" I92I, Heft I und 2 erschien eine Arbeit von SACHS, welche sowohl in der Eröffnungs-, Austreibungs-, wie vor allem Nachgeburtszeit die Hypophysenextrakte als souveränes Wehenmittel bei richtiger Indikationsstellung lobt.

Dem gegenüber fehlt es schon aus früheren Zeiten nicht an warnenden Stimmen. So erschien bereits im Jahre I9I 4 die Arbeit von BischoFf, „Hypophysenextrakt und Atonia uteri", Zentralbl. f. Gynäkol. 19I4, Nr. I5. Hierin stellt Bischöf die bisher in der Literatur uber dieses Thema veröffentlichten Arbeiten zusammen und mahnt an Hand eines Spätfalles von Atonie ( 2 Stunden p. part.) zur Vorsicht.

Ferner ist im Zentralbl. f. Gynäkol. I92I, Nr. ${ }_{5} \mathrm{I}$ eine amerikanische Arbeit referiert, welche als Schädigungen bei der Anwendung der Hypophysenextrakte anführt: ,Uterusruptur, Zerreißungen der weichen Geburtswege, Asphyxien des Kindes und intrakranielle Blutungen."

Wenn auch ich mich auf Grund eigener Erfahrungen dazu äußern đarf; so möchte ich zuerst hervorheben, daß ich in der Eröffnungszeit von der Anwendung dieser Mittel wenig Erfolg gesehen, dagegen den Exitus eines Kindes erlebt habe.

Es handelte sich um eine Viertgebärende mit frühzeitígem Blasensprung. Der Muttermund war 24 Stunden nach dem Blasensprung fünfmarkstückgroß und es trat Wehenschwäche ein. Nach Pituglandol wurden die Wehen kräftiger. Bei der dritten Wehe wurden die bis dahin regelmäßïgen Herztöne langsam, um nach der nächsten. Wehe völlig aufzuhören.
Das gleiche hat sich in zwei Fällen bei stehender Blase und PreBwehen ereignet.

Der Kopf stand auf dem Beckenboden. Hier gab ich wegen der sekundären Wehenschwäche (keine rigiden Weichteile) Pituglandol mit dem Erfolge, daß sehr kräftige Wehen einsetzten; in dem einen Fall sogar vorher ein tetanischer Krampfzustand des Uterus. Plötzlich wurden die Herżtöne schlecht, und ehe ich eingreifen konnte, waren beide Kinder tot. In einen weiteren Falle machte ich in der Austreibungszeit bei auf dem Beckenboden stehendem Kopf wegen sekundärer Wehenschwäche eine Einspritzung. Die vorher regelmäßigen Herztöne wurden nach zwei kräftigen Wehen sehr langsam. Durch sofortige Zange wurde ein schwer asphyktischesKind geboren.

Diese kurz hintereinander vorgekommenen Fälle haben mich veranlaßt, doch sehr vorsichtig bei der Anwendung der Hypophysenpräparate zu sein. Zwar habe ich die Bedeutung dieser Mittel während der Austreibungszeit in einer früheren Arbeit ,Uber Erleichterung der Geburt" anerkannt, doch ist es geboten, daß man in jedem Falle vor Anwendung ,zangenbereit" ist, um bei Gefahr sofort eingreifen zu können. Ich halte es also im allgemeinen für erforderlich, stets alles zu einer operativen Beendigung der Geburt vor Anwendung der Hypophysenpräparate vorzubereiten, um nicht im Notfalle durch Zeitversäumnis ein Kind zu verlieren. Wie sehr diese Mittel auf den Uterus wirken, erhellt am besten aus einem Fall von Spontanruptur des Uterus bei einer Zweitgebärenden mit auf dem Beckenboden stehendem Kopfe, bei der auf der Höhe einer Preßwehe, welche außerordentlich kräftig war, der Uterus plötzlich rupturierte.

Bei der Wehe war stets ein etwa fünfmarkstückgroßes Segment des Kopfes sichtbar. Trotzdem, daß dieser Fall sich außerhalb des Hospitals ereignete, hatte ich das Glück, die Frau sofort dorthin bringen lassen zu können und hatte sie 20 Minuten nach dem Unglücksfalle auf dem Operationstisch. Bei der Laparotomie zeigte sich die Hinterwand der Cervix und der Scheide längszerrissen. Placenta und Foetus lagen in der Bauchhöhle. Nach Amputation des Uterus und Vernähen der Scheiden- und Cervixwunde trat glàtte Heilung ein.

Wenn man also in der Austreibungszeit über die Bedeutung der Hypophysenextrakte als bewährtes Wehenmittel nur eine Meinung haben kann, wobei noch zu erwähnen ist, daß manchmal Pituitrin versagt, wo Pituglandol wirkt und auch umgekehrt, so ist doch bei stehender Blase, aber auch nach dem Blasensprunge und PreBwehen genau die Herztätigkeit des Kindes zu beobachten. Ich stelle hierbei zur Diskussion, ob es nicht bei stehender Blase und indizierter Anwendung der Hypophysenpräparate geboten wäre, vor deren Einspritzung die Blase zu sprengen.

Für die Nachgeburtszeit sind die Gefahren betreffs Atonie nach Gebrauch der Hypophysenpräparate in der Austreibungszeit wiederholt beobachtet und es gibt sicherlich eine Reihe Ärzte, welche direkt schon nach der Geburt deshalb prophylaktische Secalepräparate geben. Auch ich habe schwerste Atonien als Folge derAnwendung von Hypophysenpräparaten in der Austreibungszeit gesehen, während ich in keinem Falle eine besondere Wirkung dieser Mittel bei Atonia uteri oder adhärenter Placenta, wie sie veröffentlicht ist, beobachten konnte.

Zusammenfassung: In der Eröffnungszeit haben die Hypophÿsenpräparate keinerlei besondere Wirkung.

In der Austreibungszeit sind Pituglandol und Pituitrin als wehenanregende Mittel bei richtiger Indikationsstellung besonders bewährt, doch ist Vorsicht geboten bei stehender Blase und Preßwehen wegen Gefahr für das Kind. Ich halte es für zweckmäßig, vor Anwendung bei Preßwehen und stehender Blase diese zu sprengen. Wer aber dieses nicht tun will, um die Frau nicht innerlich berührt zu haben, soll stèts wegen der Géfahr für das Kind alles für eine operative Entbindung , vorbereitet halten, im besonderen soll man bei Anwendung der Hypophysenpräparate ,zangenbereit". sein.

Nach Anwendung der Hypophysenpräparate in der Austreibungszeit ist besonders auf Nachgeburtsblutungen zu achten, da Atonien häufig Folgeerscheinungen sind. Prophylaktische Secalegaben sind anzuraten.

Zur Bekämpfung von atonischen Nachblutungen haben die Hypophysenpräparate nicht die Bedeutung, wie die Secalepräparate. 